\title{
An Improved Fuzzy Rule-Based Automated Trading Agent
}

\author{
Héctor Allende-Cid ${ }^{1}$, Enrique Canessa ${ }^{2}$, Ariel Quezada ${ }^{3}$, Héctor Allende $^{\mathbf{4}}$ \\ ${ }^{1,4}$ Universidad Técnica Federico Santa María, Departamento de Informática, \\ Avda. España 1680, Valparaíso, 2390123, Chile, \\ \{vector,hallende\}@inf.utfsm.cl \\ 2 Universidad Adolfo Ibañez, Facultad de Ingeniería y Ciencias, \\ Balmaceda 1625, Viña del Mar, 3132386, Chile, \\ ecanessa@uai.cl \\ 3 Universidad Adolfo Ibañez, Facultad de Sicología, \\ Balmaceda 1625, Viña del Mar, 3132386, Chile, \\ ariel.quezada@uai.cl
}

\begin{abstract}
In this paper an improved Fuzzy Rule-Based Trading Agent is presented. The proposal consists in adding machine-learning-based methods to improve the overall performance of an automated agent that trades in futures markets. The modified Fuzzy Rule-Based Trading Agent has to decide whether to buy or sell goods, based on the spot and futures time series, gaining a profit from the price speculation. The proposal consists first in changing the membership functions of the fuzzy inference model (Gaussian and Sigmoidal, instead of triangular and trapezoidal). Then using the NFAR (Neuro-Fuzzy Autoregressive) model the relevant lags of the time series are detected, and finally a fuzzy inference system (Self-Organizing Neuro-Fuzzy Inference System) is implemented to aid the decision making process of the agent. Experimental results demonstrate that with the addition of these techniques, the improved agent considerably outperforms the original one.
\end{abstract}

Keywords: Automated Trading Agents, Fuzzy Rule-based Agents.

\section{Introduction}

In recent years the existence of many digital mediated tasks that present participants with complex strategic decisions has been more obvious. The agent paradigm has led to decisions that involve a significant interaction between them. The importance of financial trading and electronic commerce can not be ignored, not only for the negotiation and trading involved, but also because of other commercial operations, such as matchmaking, resource finding, advertising, recommendation, contracting and executing transactions [20]. Online markets have set the spotlight in the interest to automate some tasks. There are many reasons why it could be beneficial to keep exploring the automation of financial markets. One advantage is cost saving from automating some functions of the existing noncomputational markets. Other could be the exclusion of human behaviour from trading agents. It is known that humans often make emotional decisions, based on biased perceptions of the world. This aspect may be more detrimental when agents need to make fast decisions. Thus, rationalizing the behaviour of agents could be most of the time beneficial from many points of view. Another drawback of human trading agents is that nowadays, the amount of information that is generated is so large, and in such short periods of time, that it is necessary to have agents that can process vast amounts of data and make decisions in seconds, or even in shorter time. This is why we need to have agents with high computational capacity and very fast automated responses. Various works have been proposed in this field, for example $[12,14,15,16]$, where the authors propose and compare automated trading agents that deal with stock trading and supply chain management, among other applications. Additionally, there are many trading agent competitions [1,17], that are designed to promote and encourage high quality research regarding the trading agent problem.

In the last decades fuzzy logic has been an active area of research, many research efforts have been focused on fuzzy modelling and control issues based on the Takagi-Sugeno (TS) fuzzy model $[4,6,9,10]$, which is described by fuzzy IF-THEN rules. Also the inclusion of this approach to many computational intelligence models has improved them by providing approximate reasoning to models that are generally probabilistic or “crisp” [2, 5,8, 21]. 
The structure of this work is organized as follows: in the next section, we briefly discuss the fundamentals of Futures Market, present the U-Mart Project and explain the Fuzzy Rule-based trading agent. In section 3 we present our proposal. Then we give some experimental results to show the performance of the proposed agent in section 4. Finally, in the last section, we present the concluding remarks and we delineate some future work.

\section{Background and State of the Art}

\section{Futures Market}

Futures trade is a trade to buy or sell goods at a certain point of time in the future (due date). The price of futures goods is determined as an actual market price (spot price) at the due date and is unknown before that date.

Exchange transactions consist of the following three aspects:

- Agreement (Decision to buy or sell)

- Delivery (deliver the commodities or securities from seller to buyer)

- Settlement (handling of money)

Transactions simultaneously conducting the above three aspects are called spot tradings. In futures trading only the agreement is made at present time: delivery and settlement are conducted at a future date.

More precisely, futures trading is a transaction in which a seller and buyer agree to turn over a certain product or security at a given future due date, with the price determined at present time (this is called 'a contract'). The price and volume of the transaction are determined at present time, whereas the product is not delivered until the settlement date.

In futures market there are three types of participants. Hedgers are participants who desire to avert price volatility risk. Arbitragers try to use the profit margins of the price difference among markets to make a profit. In this particular work the automated trading agent will be a speculator, a trader who predicts the change in a future price and aims at gaining a profit with his / her prediction.

\section{U-Mart Project}

U-Mart is a project that creates a virtual futures market on a stock index using a computer or network of computers in order to promote on-site training, education, and even economic research [18]. This software is aimed at students, teachers, and researchers, as well as individuals who are interested in stock and index futures trading.

The participants in U-Mart (Human or Machine Agents) are given a certain amount of funds, so that they can make decisions whether to buy or sell, and how much. The number of days and sessions per day are given in advance. In each session participant traders have to decide whether to buy or sell, and the amount. After all the trading sessions are concluded the software calculates the total funds remaining for each agent. If that amount is larger than the initial one, the Agent has made a profit and can be considered successful. For more details please visit [18].

In Figures 1 and 2, we can see a simple diagram of the trade that is conducted by using this framework and the GUI of U-Mart.

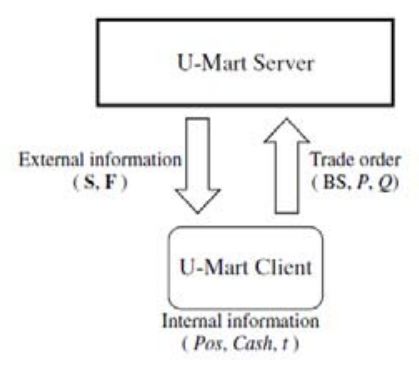

Figure 1. A view of the trade in U-Mart [13]

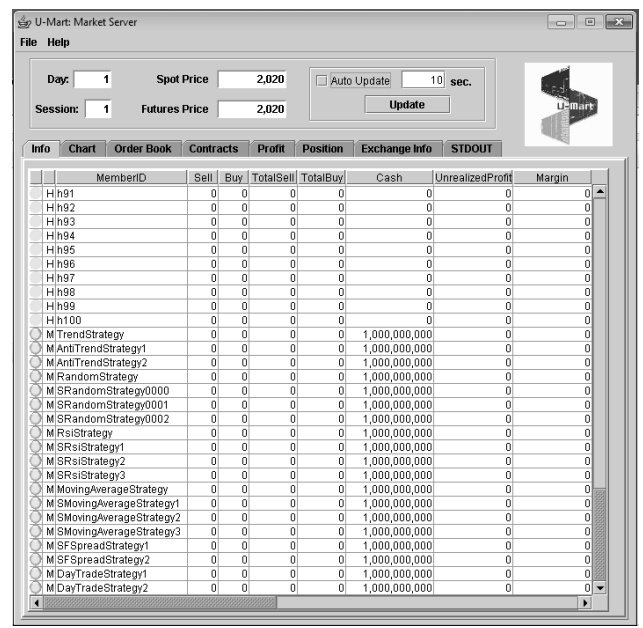

Figure 2. A snapshot of U-Mart Server 


\section{Fuzzy Rule-Based Trading Agent}

In [13], the authors present a fuzzy rule-based agent for trading in futures market. Implemented within a fuzzy rule-based system paradigm, the machine agent determines whether to buy or sell futures spot based on both spot and futures time series. The fuzzy rule-based agent is fine-tuned to maximize the amount of profit it can get.

\section{A. Problem formulation and fuzzy If- Then rules.}

In the virtual futures market described in the latter subsection, the time series of both spot and futures prices are available to the agent. The problem of the futures market described in this paper can be viewed as a two-class pattern classification problem with 3dimensional inputs. Let $S=\left(s_{1}, \ldots, s_{m}\right)$ and $F=\left(f_{1}, \ldots, f_{m}\right)$ be the Spot and Futures Time Series respectively (see Figure 3). The fuzzy rules used in [13] are the following:

$R_{j}$ : If $x_{1}$ is $A_{1}$ and $x_{2}$ is $A_{2}$ and $x_{3}$ is $A_{3}$ then Buy with $q_{j 1}$ and Sell with $q_{j 2}, j=1, \ldots, N$.

Where $R_{j}$ is the label of the $j$-th fuzzy if-then rule. $A_{1}, A_{2}, A_{3}$ are antecedent fuzzy sets, $q_{j 1}$ and $q_{j 2}$ are values of the fuzzy if-then rules,

$x_{1}=s_{m}-f_{m}$,

$x_{2}=\sum_{i=3}^{5}\left(s_{m-i}-f_{m-i}\right) / 3$,

$x_{3}=\sum_{i=8}^{10}\left(s_{m-i}-f_{m-i}\right) / 3$,

The antecedent fuzzy set membership functions used in [13] are triangular and trapezoidal (see Figure 4).

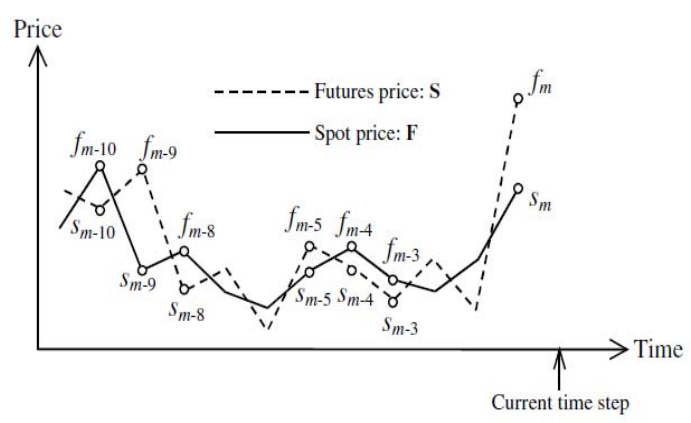

Figure 3. Time Series of both Spot and Futures Prices [13]

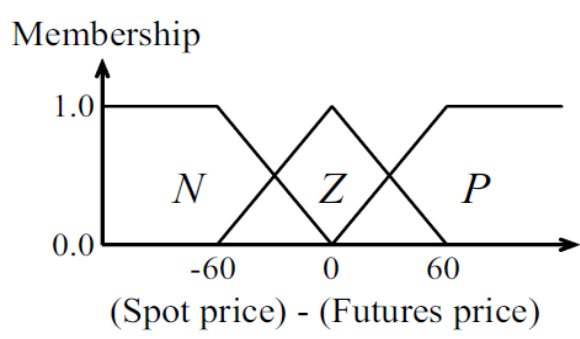

Figure 4. Membership functions used in the Fuzzy Trading Agent [13]

\section{B. Fuzzy inference and decision making of the agent}

Let us consider that at a particular time we have already calculated the three input variables $x_{1}, x_{2}$ and $x_{3}$ for making the next decision (buy or sell and the respective quantity). We calculate now the values for the variables $Q_{B u y}$ and $Q_{\text {Sell, }}$ using the following equation:

$Q_{Y}=\frac{\sum_{j=1}^{N} \mu_{j}(\tilde{x}) \times q_{j Y}}{\sum_{j=1}^{N} \mu_{j}(\tilde{x})}$,

where $Y \in$ Buy, Sell , $\tilde{x}=x_{1}, x_{2}, x_{3}, \mu_{j}(\cdot)$ is a multiplication operator ( $\mathrm{T}$-norm product) associated with $R_{j}$.

After calculating $Q_{B u y}$ and $Q_{\text {Sell }}$, the agent makes the decision on whether it buys or sells the futures stock according to the following decision rule:

- If $Q_{B u y}>Q_{\text {sell, }}$ the agent buys the futures stock.

- Else if $Q_{\text {Buy }}<Q_{\text {Sell, }}$, the agent sells the futures stock.

- Otherwise the agent keeps the same decision made at the previous time step.

\section{Decision making on the limit price and quantity}

The authors in [13] assume that the agent tries to optimize the decision making only on whether it buys or sells the futures stock. Thus, fuzzy if-then rules in the fuzzy rulebased system have weights corresponding to only Buy or Sell. In the above mentioned Virtual Futures Market U-Mart, each agent must determine not only whether it should buy or sell but also the quantity of the transaction. 
After determining whether to buy or sell futures stock by the procedure described in the last subsection, the other information necessary for the trade order is the limit price $P$ (price at which the agent is willing to buy/sell the stocks) and the quantity.

$$
P= \begin{cases}f_{m}-15, & \text { if decision is Buy } \\ f_{m}+15, & \text { if decision is Sell }\end{cases}
$$

$f_{m}$ is the latest spot price. That is, the limit price is determined based on the futures price at the previous time step. Then the quantity $Q$ of the trade is determined according to the values obtained for $Q_{B u y}$ and $Q_{\text {sell, }}$, respectively.

$$
Q= \begin{cases}Q_{\text {Buy }} \times 100, & \text { if decision is Buy } \\ Q_{\text {Sell }} \times 100, & \text { if decision is Sell }\end{cases}
$$

\section{On-line learning of fuzzy If-Then rules (reinforcement learning)}

The authors specify an equation to modify $q_{Y j}$ depending on the results the agent is obtaining at a current run of an U-Mart experiment [13]. Although they never claim to be applying reinforcement learning, they penalize in some manner incorrect decisions and reinforce correct ones. For example, let us assume that the agent has already made a decision on whether it buys or sells the futures index. At the next time step, the agent is given a new value of the time series data of both spot and futures prices. In [13], the authors evaluate the agent's decision based on the latest prices $s_{m+2}$ and $f_{m+1}$ and the evaluation values $Q_{\text {Buy }}$ and $Q_{\text {Sell }}$ that were previously calculated:

- If $Q_{\text {Buy }}>Q_{\text {Sell }}$, and $s_{m+1}>f_{m+1}$, then the decision at the previous time step is evaluated as successful agent buys the futures stock.

- Else if $Q_{\text {Buy }}>Q_{\text {Sell, }}$, and $s_{m+1}>f_{m+1}$, the decision is also considered successful.

- - Otherwise the decision at the previous time step is evaluated as unsuccessful.

That is, the evaluation of the decision is based on the absolute price difference between the spot and futures price. If the agent's decision is Buy, the evaluation of the decision is successful only if the spot price is higher than the futures price since in this case it is expected that the agent can gain some profit at the final settlement. On the other hand, if Sell is chosen, the evaluation of the decision is successful only if the spot price is lower than the futures price. The evaluation described above is used for updating the weights of fuzzy if-then rules. The main idea of the weight update is that the weights of those fuzzy if-then rules that contribute to the successful decision making are increased while those fuzzy if-then rules that are responsible for unsuccessful decision making are decreased. The update rule of the weights is:

$$
\begin{aligned}
& q_{j Y}^{\text {new }}=\left\{\begin{array}{lr}
q_{j Y}^{\text {old }}+\alpha \cdot\left(1-q_{j Y}^{\text {old }}\right) \mu(\tilde{x}), & \text { if succesful } \\
q_{j Y}^{\text {old }}+\alpha \cdot q_{j Y}^{\text {old }} \mu(\tilde{x}), & \text { otherwise }
\end{array}\right. \\
& Y \in \text { Buy, Sell, } \quad j=1, \ldots, N
\end{aligned}
$$

The authors examined the weights of each fuzzy if-then rule to select a small number of fuzzy if-then rules with strong contrast between weights for Buy and Sell. From a total number of $27\left(=3^{3}\right)$ rules they manually selected 5 fuzzy if-then rules which are presented in Table 1.

Table 1. Optimal weights

\begin{tabular}{|l|l|l|l|l|l|}
\hline No. & $x_{3}$ & $x_{2}$ & $x_{1}$ & $q_{j 1}$ & $q_{j 2}$ \\
\hline 1 & $\mathrm{~N}$ & $\mathrm{~N}$ & $\mathrm{~N}$ & 0.598 & 0.086 \\
\hline 2 & $\mathrm{~N}$ & $\mathrm{Z}$ & $\mathrm{P}$ & 0.778 & 0.318 \\
\hline 3 & $\mathrm{~N}$ & $\mathrm{P}$ & $\mathrm{N}$ & 0.546 & 0.870 \\
\hline 4 & $\mathrm{P}$ & $\mathrm{Z}$ & $\mathrm{Z}$ & 0.573 & 0.800 \\
\hline 5 & $\mathrm{P}$ & $\mathrm{P}$ & $\mathrm{P}$ & 0.924 & 0.141 \\
\hline
\end{tabular}

\section{Proposal}

In the present work we propose an improvement to the fuzzy trading agent presented in the latter section. Since the results obtained by using the strategy proposed by [13] does not achieve considerably higher profits than the default agents included in the U-Mart Project [6], we think that there is room for improving the strategy developed by [13]. Thus, we will apply the following techniques for enhancing it:

- Change the Fuzzy Membership functions from triangular and trapezoidal to smoother ones, coming from the exponential and sigmoidal family.

- Search for the most influential lags in the Spot and Futures Time Series to try to 
enhance the fuzzy if-then rules stated in equations (1), (2) and (3).

- Add a forecasting model, to try to predict values for the time series, and thus improve the agent's decisions.

\section{Gaussian and Sigmoidal membership functions}

One of the proposals is to change the original membership functions (triangular and trapezoidal) to Gaussian and Sigmoidal membership functions (see figure 5). Smooth membership functions are continuous in all the domain, an important factor when optimizing a function with gradient-based methods [7].

$$
f(x ; \sigma, c)=e^{\frac{-(x-x)^{2}}{2 \sigma^{2}}}
$$

where $\sigma=30$ and $c=0$

$$
f(x ; a, c)=\frac{1}{1-e^{-a(x-c)}}
$$

where $a_{1}=-0.1, c_{1}=-40, a 2=0.1$ and $c 2=40$.

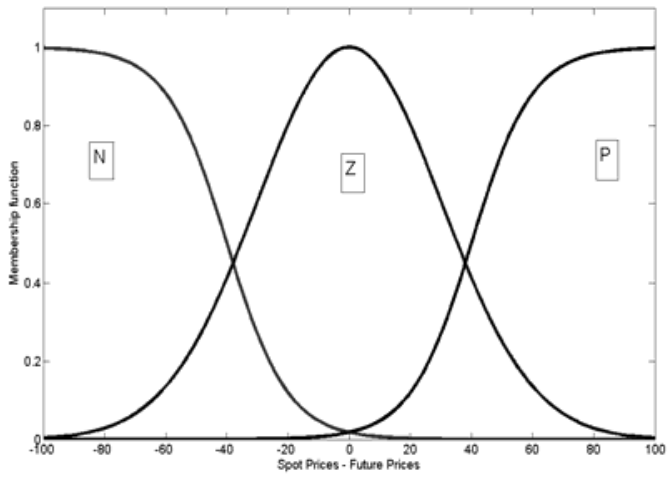

Figure 5. Proposed Membership Functions

\section{Detect relevant Time Series Lags}

A common feature of all time series is that the current value is dependent on the previous ones, and a dynamic model is required for a proper description of the real system that generates the observations. In this way, an important problem in forecasting procedures is the empirical model building. This involves the selection of an appropriate set of lagged values of the time series that will be used as independent variables to be able to build an improved forecasting model. The aim of this proposal is to simultaneously identify and estimate the nonlinear autoregressive time series of both the future and spot prices using a flexible neuro-fuzzy model [19]. The model provides a self organizing and incremental mechanism to the adaptation process of the neuro-fuzzy model. The self organization mechanism searches for a suitable set of premises and consequents to enhance the time series estimation performance, while the incremental method selects influential lags in the model description. With this algorithm we searched for the most relevant lags of the spot and futures prices time series. With the sequential Neuro-Fuzzy Autoregressive Model we found 3 relevant lags.

$$
\begin{aligned}
& x_{1}=s_{m}-f_{m}, \\
& x_{2}=s_{m-2}-f_{m-2}, \\
& x_{3}=s_{m-3}-f_{m-3},
\end{aligned}
$$

Having found these relevant lags we can confidently say that these variables model the behaviour of the time series with a smaller Mean-Squared Error (MSE) than the original lags presented in [13] (see expressions (1) to (3)).

\section{Neuro-fuzzy inference model}

To aid the strategy of the agent with a more elaborate forecasting method a neuro-fuzzy inference system [3] was added to its strategy. A neuro-fuzzy inference system is functionally equivalent to a Takagi-sugeno fuzzy inference system. The model presented in [3] is an adaptive learning algorithm that automatically designs a neuro-fuzzy model, called SONFIS (Self-Organizing NeuroFuzzy Inference System) (see figure 6). This constructive learning algorithm attempts to identify the fuzzy rule base and their parameters of the Jang's ANFIS model [11] based on an architectural self-organization mechanism with a data-driven approach. The proposed training algorithm self-organizes the model premises by using adding, merging and splitting operations. Each fuzzy-rule competes to learn specific training patterns and, to accomplish this task; the algorithm can either add new rules, merge correlated rules or split existing rules with unsatisfactory accuracy performance.

The self-organization learning procedure consists of two stages. In the first stage we 
construct a base model with a predefined number of nodes and we estimate the parameters using gradient-based method and Least Square Estimation (LSE). During the second stage we proceed to apply three types of operators: Grow Net (GrowNet), Split Membership Functions (SplitNet) and Vanish Membership Functions (VanishMF). These operators are applied iteratively until the net self organizes and stabilizes satisfying a user's performance criterion. Based on the forecast of this model, if the price forecasted in one step is higher than the price at a present time, combined with the previous strategy, the agent buys, and if it is lower otherwise. With the inclusion of this information we show in the next section that the profit increases considerably comparing to the other agents.

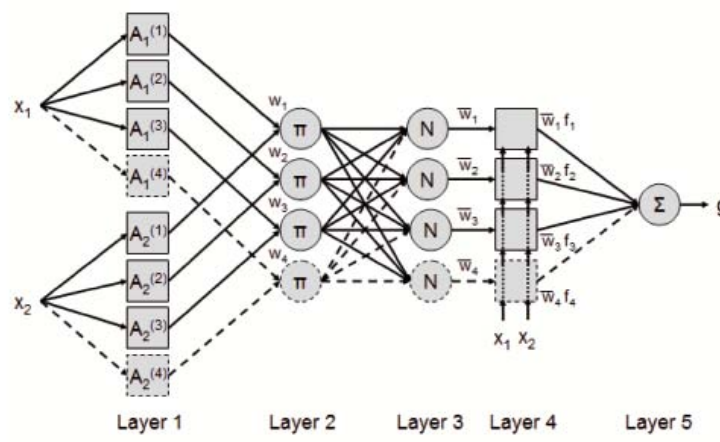

Figure 6. The SONFIS model

\section{Experimental Results}

As was explained before the experimental runs were performed using the U-Mart Project platform [6]. The spot time series used are the so-called J30, a well-known stock index of thirty selected Japanese firms, which is calculated by Mainichi Newspapers Co., Ltd. and the umie2005reversal, a time series included in the UMIE2005 Trading Agent competition.

The experimental runs for both time series were 10 and we tested the new strategy using the denominated sliding window approach. The total number of observations of the J30 time series is 480 . But since the agents are trading in the market only for 30 days and 8 sessions per day, the number of observations necessary is only 240 . We generated 10 different data sets of 240 observations each with this approach. The results can be seen in Table 2.The total number of observations of the umie2005reversal time series is 361. The number of days for which the agent trades is 20 and 4 sessions per day. So the number of observations for each run is only 80 . We generated also 10 different data sets.

All of the agents start with 1.000.000 Yens. They have to make decisions whether to buy or sell and the quantity, and after 30 days of trading, the total profit is calculated. As can be seen in Table 2 the agent that has the largest profit is the modified fuzzy rule-based trading agent. The modified agent exceeds by almost $30 \%-40 \%$ the amount of profit of the other agents, but has a larger standard deviation. Nevertheless the difference is statistically significant. The t-value obtained by comparing the profit obtained by the original fuzzy strategy (FuzzyStrategyOrig) and the modified one (FuzzyStrategyProposal) was $t_{0}=8.0121$, $(\mathrm{df}=18)$, with $p$-value $=2.41 \times 10^{-7}$, which shows that those results are statistically different. In Table 3 we can see the results of our proposal compared with the same agents used in the first experiment. In this experiment we can observe that our proposal again obtains the best profit comparing with the other agents. The difference between them and out proposal is also statistically significant.

Table 2. Results of 10 Experimental Runs J30 Dataset

\begin{tabular}{|l|l|l|}
\hline Agents & Mean (Yens) & Std. Dev. \\
\hline TrendStrategy & $990.858,56$ & $23.322,35$ \\
\hline AntiTrendStrategy1 & $961.897,00$ & $27.551,80$ \\
\hline AntiTrendStrategy2 & $978.190,78$ & $30.435,16$ \\
\hline RandomStrategy & $954.468,89$ & $47.147,44$ \\
\hline SRandomStrategy0000 & $952.133,56$ & $27.001,75$ \\
\hline SRandomStrategy0001 & $957.181,89$ & $27.131,95$ \\
\hline SRandomStrategy0002 & $980.511,89$ & $20.886,20$ \\
\hline RsiStrategy & $994.525,33$ & $39.944,30$ \\
\hline SRsiStrategy1 & $986.529,33$ & $20.705,67$ \\
\hline SRsiStrategy2 & $991.309,44$ & $15.749,20$ \\
\hline SRsiStrategy3 & $987.399,67$ & $20.466,56$ \\
\hline MovingAverageStrategy & $1.002 .525,00$ & $5.321,17$ \\
\hline SMovingAverageStrategy1 & $1.001 .327,11$ & $5.860,89$ \\
\hline SMovingAverageStrategy2 & $1.002 .773,78$ & $5.657,02$ \\
\hline SMovingAverageStrategy3 & $1.001 .681,33$ & $4.464,19$ \\
\hline SFSpreadStrategy1 & $924.116,89$ & $204.687,93$ \\
\hline SFSpreadStrategy2 & $980.626,44$ & $17.831,10$ \\
\hline DayTradeStrategy1 & $938.724,00$ & $28.807,90$ \\
\hline DayTradeStrategy2 & $931.021,56$ & $18.554,41$ \\
\hline FuzzyStrategyOrig & $1.010 .482,44$ & $12.797,10$ \\
\hline FuzzyStrategyProposal & $\mathbf{1 . 4 0 5 . 0 4 8 , 4 4}$ & $137.273,69$ \\
\hline
\end{tabular}




\section{Conclusions and Future Work}

The results obtained by the modified fuzzy rule-based agent were good. Thus, we can conclude that the addition of more complex algorithms to the strategy of the agent was successful. However further experimentation is necessary to validate our proposal using Spot and Futures Time Series of different commodities or securities. Additionally, note that in our experiments only one agent was using our strategy. Thus, it would be interesting to simulate trading sessions with many of our agents and see whether we still obtain higher profits compared with those gained by other agents.

Table 3. Results of 10 Experimental Runs umie2005reversal Dataset

\begin{tabular}{|l|l|l|}
\hline Agents & Mean (Yens) & Std. Dev. \\
\hline TrendStrategy & $984.136,33$ & $29.075,50$ \\
\hline AntiTrendStrategy1 & $915.437,66$ & $88.614,43$ \\
\hline AntiTrendStrategy2 & $924.362,68$ & $86.204,45$ \\
\hline RandomStrategy & $961.996,76$ & $55.277,61$ \\
\hline SRandomStrategy0000 & $1.064 .308,29$ & $86.619,24$ \\
\hline SRandomStrategy0001 & $1.072 .291,12$ & $82.175,58$ \\
\hline SRandomStrategy0002 & $1.060 .296,48$ & $83.608,16$ \\
\hline RsiStrategy & $947.709,85$ & $96.288,87$ \\
\hline SRsiStrategy1 & $987.061,71$ & $9.178,08$ \\
\hline SRsiStrategy2 & $1.005 .543,25$ & $8.193,71$ \\
\hline SRsiStrategy3 & $1.007 .563,44$ & $11.003,37$ \\
\hline MovingAverageStrategy & $991.970,85$ & $8.126,18$ \\
\hline SMovingAverageStrategy1 & $1.010 .782,48$ & $8.512,99$ \\
\hline SMovingAverageStrategy2 & $1.012 .827,82$ & $12.342,24$ \\
\hline SMovingAverageStrategy3 & 1.011 .531 & $8.954,61$ \\
\hline SFSpreadStrategy1 & 1.065 .002 & $62.548,72$ \\
\hline SFSpreadStrategy2 & $1.064 .380,72$ & $88.744,87$ \\
\hline DayTradeStrategy1 & $960.740,85$ & $40.331,36$ \\
\hline DayTradeStrategy2 & $952.059,96$ & $38.428,41$ \\
\hline FuzzyStrategyOrig & $1.064 .391,55$ & $15.993,78$ \\
\hline FuzzyStrategyProposal & $\mathbf{1 . 2 8 1 . 8 4 7 , 7 7}$ & $55.424,22$ \\
\hline
\end{tabular}

\section{Acknowledgements}

This work was supported by the following research grants: Fondecyt 1110854 and DGIP-UTFSM.

\section{REFERENCES}

1. A. for Trading Agent Research, Online. Available: http://tradingagents.org, September, 2010.
2. AI-BING, J., P. JIA-HONG, L. SHUHUAN, S. JIAN-PING, Support Vector Machine for Classification Based on Fuzzy Training Data, Machine Learning and Cybernetics, 2006 International Conference, 13-16 August 2006, pp. 1609-1614.

3. ALLENDE-CID, H., A. VELOZ, R. SALAS, S. CHABERT, H. ALLENDE, Self-organizing Neuro-fuzzy Inference System, in Congreso Iberoamericano de Reconocimiento de Patrones, 2008, pp. 429-436.

4. BENREJEB, M., D. SOUDANI, A. SAKLY, P. BORNE, New Discrete TSK Fuzzy Systems Characterization and Stability Domain, International Journal of Computers, Communications \& Control, Agora University Editing House - CCC Publications, vol. 1, no. 4, 2006, pp. 9-19.

5. CHEN, B., L. L. HOBEROCK, A Fuzzy Neural Network Architecture for Fuzzy Control and Classification, Neural Networks, 1996, IEEE International Conference, vol. 2, 3-6 June 1996, pp. 1168-1173.

6. DIEULOT, J. Y., Fuzzy Control and Estimation Using Model Inversion, Studies in Informatics and Control Journal, ICI Publishing House, vol. 13, no. 3, 2004, pp. 153-160.

7. GUELY, F., P. SIARRY, Gradient Descent Method for Optimizing Various Fuzzy Rules Bases, in Second IEEE International Conference on Fuzzy Systems, 1993, pp. 1241-1246.

8. GYEONGYONG, H., P. GADER, Fuzzy SVM for Noisy Data: A Robust Membership Calculation Method, Fuzzy Systems, 2009. FUZZ-IEEE 2009, IEEE International Conference, 20-24 August 2009, pp. 431-436.

9. HAFAIFA, A., K. LAROUSSI, F. LAAOUAD, Robust Fuzzy Fault Detection and Isolation Approach Applied to the Surge in Centrifugal Compressor Modelling and Control, International Journal of Fuzzy Information and Engineering, Springer, vol. 2 no. 1, 2010, pp. 49-73. 
10. HAFAIFA, A, F. LAAOUAD, K. LAROUSSI, Fuzzy Modelling and Control for Detection and Isolation of Surge in Industrial Centrifugal Compressors, Journal of Automatic Control, University of Belgrade, vol. 19, no. 1, 2009, pp. 19-26.

11. JANG, J.-S. R., ANFIS: Adaptivenetwork-based Fuzzy Inference System, IEEE Transactions on Systems, Man and Cybernetics, vol. 23, no. 3, 1993, pp. 665-685.

12. KEARnS, M., L. ORTIZ, The PennLehman Automated Trading Project, IEEE Intelligent Systems, IEEE Computer Society, 2003.

13. NAKASHIMA, T., T. ARIYAMA, H. KITANO, H. ISHIBUSHI, A Fuzzy Rule-Based Trading Agent: Analysis and Knowledge Extraction, Computational Intelligence Modelling and Prediction Book, Springer, 2005.

14. PREIST, C., Economic Agent for Automated Trading, Technical Report, Hewlett Packard Labs, 1998.

15. SHERSTOV, Y, P. STONE, Three Automated Stock-Trading Agents: A Comparative Study, Agent Mediated Electronic Commerce, Springer, 2005.
16. SNARSKA, M., J. KRZYCH, Automatic Trading Agent: RMT based Portfolio Theory and Portfolio Selection, Acta Physica Polonica B, vol. 37, no. 11, 2006, pp. 3145-3160.

17. T.A.Competition, Online. Available: http://www.sics.se/tac, September, 2010.

18. U. Systems, Online. Available: http://www.u-mart.org, September 2010.

19. VELOZ, A., H. ALLENDE-CID, R. SALAS, H. ALLENDE, A Flexible Neuro-fuzzy Autoregressive Technique for Non-linear Time Series Forecasting, in Knowledge and Engineering Systems, 2009, pp. 22-29.

20. WELLMAN, J., Online Marketplaces, in Practical Handbook of Internet Computing, CRC Press, 2004.

21. ZHENG, P., Q. KEYUN, X. YANG, Dynamic Adaptive Fuzzy Neuralnetwork Identification and Its Application, Systems, Man and Cybernetics, IEEE International Conference, 5-8 October 2003, vol. 5, pp. 4974-4979. 